\title{
Filter Algorithm Simulation and Logical Structure of Spectral Analysis in New Media Hardware
}

\author{
HongXia $\mathrm{Hu}^{1}$, Shi Feng ${ }^{1}$
}

${ }^{1}$ Department of physical Education, Nanchang Institute of Technology, Nanchang, 330099, China

hongxia_hu@yeah.net

Keywords: Mapping relations, Low pass filter, Digital, New media, Component, 3D scanning.

Abstract. In order to realize the art analysis of aerobics continuous movement, based on WPF and JAVA heterogeneous platforms, and combined with modern digital media technology, this paper proposes the video image analysis of modular design concept. Through the analysis of the visualization business process for the 3D aerobics scanning image, this paper divides the basic functions of visualization platform from the logically, and realizes the function. Through the aerobics movement display platform, we can get the map relationship of gymnastics artistic movement analysis, successfully filtering disorganized action image, which provides a new computer method for the study o of aerobics art.

\section{Introduction}

High performance digital signal processor is a special digital signal processor, which is very suitable for embedded image processing. The aerobics movement elements analysis needs to combine with the continuous of images to present the beauty of art, so it has high requirement on the real-time and efficiency of image processing [1,2]. The development foundation of digital media arts is digital media technology. In the field of artistic creation, digital media technology is not only the significance of tools, technology aesthetic, artistic or idea have made skill fusion as a mixture of nature cannot distinguish, it is also involved in auxiliary artist conception and imagination [3-5]. Digital media art has caused a lot of digital arts, such as Fluxus art, avant-garde film, video art, digital art and net art, their development and high technology have close relationship of interdependence. In this paper, the digital multimedia technology has applied to the aerobics art movement in the process of analysis, which realizes the analysis of image aerobics art [6]. The overall design framework is in Figure 1.

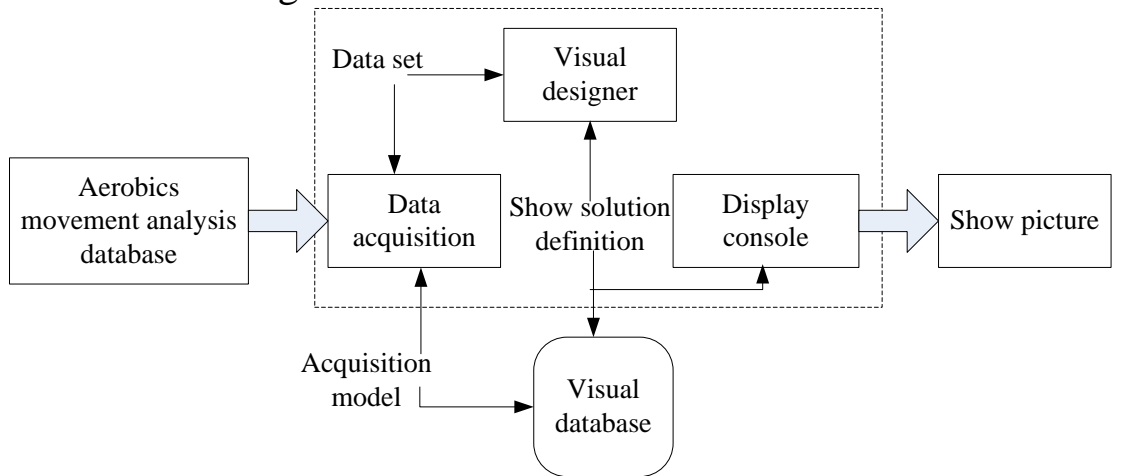

Fig.1: Analysis of the aerobics movement visual art

Figure 1 show the overall design diagram of aerobics movement visual art analysis, the scheme is mainly based on visualization technology, to carry on the aerobics movement data analysis. The visualization database is the core of information visualization platform, in which the system data storage and retrieval, case definition storage and retrieval as well as console configuration information are stored in a visual database [7,8]. However data acquisition, graphics and display console are based on visual database as a bridge to carry on data sharing. 


\section{The Mathematical Modeling and Algorithm Design of Aerobics Action Art Analysis}

For the process of aerobics art movement analysis, it mainly uses 3D camera technology to scan the aerobics movement, the higher aerobics movement art color image will be marked, and then the use of filter will be subjected to the action of filtering [9]. Through the action element analysis technique, the image is segmented, and each frame of image can be clustered. The clustering method used in this paper is the Kmeans clustering algorithm, and the clustering center can be expressed as:

$$
D=\frac{\sum_{j=1}^{m} \sum_{a_{i} \in b_{i}} K\left(a_{i}, b_{i}\right)}{\sum_{j=1}^{m}\left|B_{i}\right|} .
$$

Among then, $K$ is distance function; $m$ indicates the number of computing image cluster. This process is coupled to a nonlinear process, and it wants to filter the aerobics image information by means of fractional calculus [10]. Firstly the definition of fractional calculus, hypothesis $f(x)$ takes $x$ independent variable as function, the range of $x$ is $\left[k_{1}, k_{2}\right]$, the expression can be written as

$$
{ }_{k 1} D_{k 2}{ }^{a} f(x)=f^{(a)}(x) .
$$

Among then, $w$ represents the frequency, when $a>0$, it is the representation of integer order calculus; when $a<0$, it represents fractional calculus, in which the Fourier transform is

$$
F(w)=\int_{-\infty}^{+\infty} f(x) e^{-j w x} d x
$$

Based on the nature of the $\mathrm{Fu}$ Liye transform, the $n$ order derivatives of $f(x)$ should satisfy $f^{(n)}(x) \Leftrightarrow(j w)^{n} F(w)$, and then it is extended to fractional order that can be obtained

$$
f^{(a)}(x) \Leftrightarrow D^{a} F(w)=(i w)^{a} F(w) .
$$

Laplasse changes are the most commonly used analysis methods of invariant system analysis in continuous spectrum, assuming that complex frequency is $x=\alpha+i \omega$, integral limit is 0 , and then the signal processing expression of aerobics image is

$$
\begin{aligned}
& L[f(t)]=F(x)=\int_{0}^{\infty} f(t) e^{-x t} d t \\
& L[F(x)]=f(t)=\frac{1}{2 \pi i} \int_{\sigma-i \infty}^{\sigma+i \infty} F(x) d x .
\end{aligned}
$$

According to the Laplasse transform fractional integral form, it can be written as

$$
{ }_{k 1} D_{t}^{-a} f(t)=\frac{1}{\Gamma(a)} \int_{0}^{t} f(x)(t-x)^{a-1} d x=t^{a-1} * f(t) .
$$

After merging the frame, the aerobics movement factors decomposition model can be obtained, as shown in the formula (5)

$$
H^{p}(t)=\bigcup_{r=1}^{K} T_{r}^{p}(t), p=1,2, \cdots, 5 \quad 1 \leq t \leq l .
$$

Among them, $T$ is sample length; $l$ is difference length. Through the multi dimension visualization design, we can get the effects of aerobics different artistic, including the programming algorithm of dimension entity definition is as follows [11,12]:

using System.Runtime.Serialization;

using StateGrid.Data;

using VisualDiagram.Data;

namespace StateGrid.Acquisition.Data

$\{[$ DataContract $($ Name $=$ "Demension") $]$ 
public class Demension : EntityBase

\{private string _id;

private string _value;

private DemensionType _demensionType;

[DataMember(Name = "id")]

public string Id

\{get $\{$ return_id; \}

set

\{if (_id != value)

\{_id = value;

OnPropertyChanged("Id");\}

\}\}\}

\section{Research on New Media Aerobics Art based on Digital}

In order to verify the effectiveness and reliability of calculus low-pass filtering algorithm designed in section second, this paper takes aerobics image process as an example, combined with the virtual interactive environment of modern digital new media, the use of 3D tomography scanning technology expands the artistic analysis of aerobics movement, including virtual interactive scenes are shown in Figure 2.

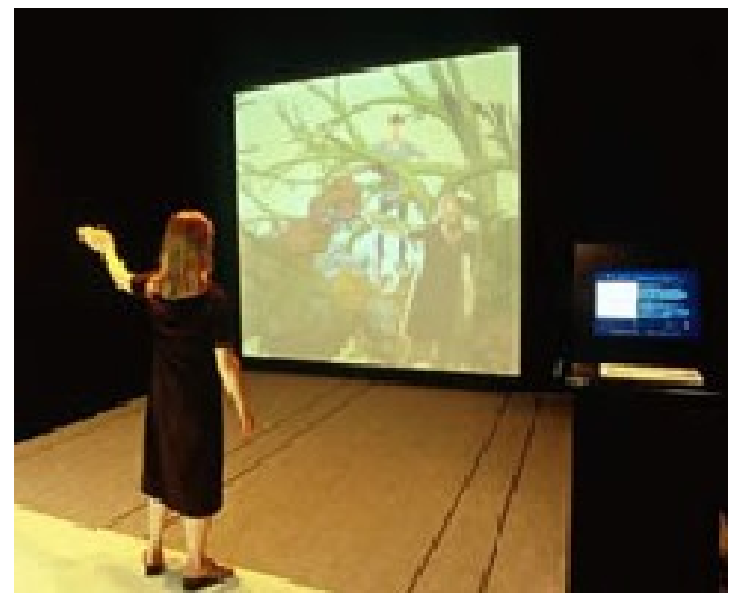

Fig.2: Aerobics interactive scene

Figure 2 represents the aerobics movement design of virtual interactive scene, the system provides a more flexible and self interactive design for the audience, the user can input a random text, this text becomes aerobics code, and then through artist design "text to image editor" into three-dimensional autonomous behavior action, aerobics code and user interaction will determine the body form, behavior change, survival condition as well as mutual influence and role of the artistic movements.

Table 1: Aerobics 3D image acquisition

\begin{tabular}{lll}
\hline Field name & Value type & Description \\
SchemaCode & varchar & Schema logo \\
\hline recodeTime & Date & time \\
GatherTime & Date & 3D photography time \\
ColumnValue1 & Integer & 1 \\
ColumnValue2 & Integer & 2 \\
\hline
\end{tabular}

Table 1 shows the record sheet of the aerobics 3D image acquisition, wherein ColumnValue1, ColumnValue2 and other field name in the data acquisition process are wrote different values according to the configuration associated with different, and then the $N$ time data collection carries out correlation analysis, we can gain good artistic image effect to compare, in which the artistic effect different font size is shown in Figure 3. 


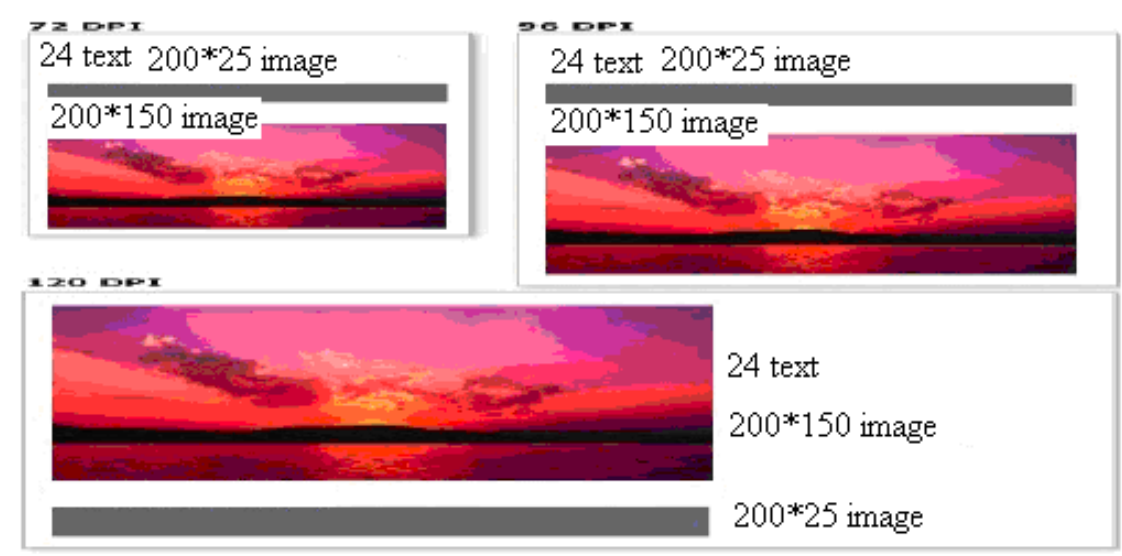

Fig.3: Comparison of WPF DPI art effect

As shown in Figure 3, it represents the effects contrast of the WPF different artistic, WPF supports to automatic expansion, image and text appropriate expansion through the pixel will be device independent as the main units of measurement, visualization platform needs to meet the different resolution to construct different monitoring surface.

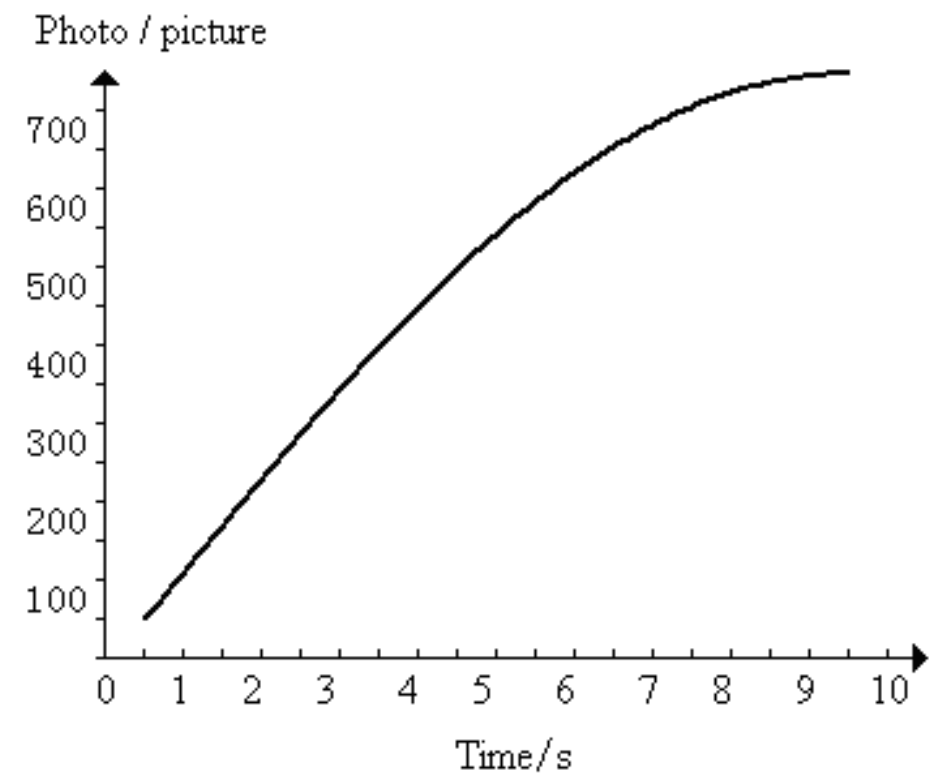

Fig.4: Data transmission curve

Figure 4 shows the scanning of the aerobics image using 3D photography, we can get the charge of curve the aerobics image frames and time in the period of time [13-15]. It can be seen from the chart that the transmission process of aerobics image is relatively stable, and it is consistent with the requirements of data transmission platform.

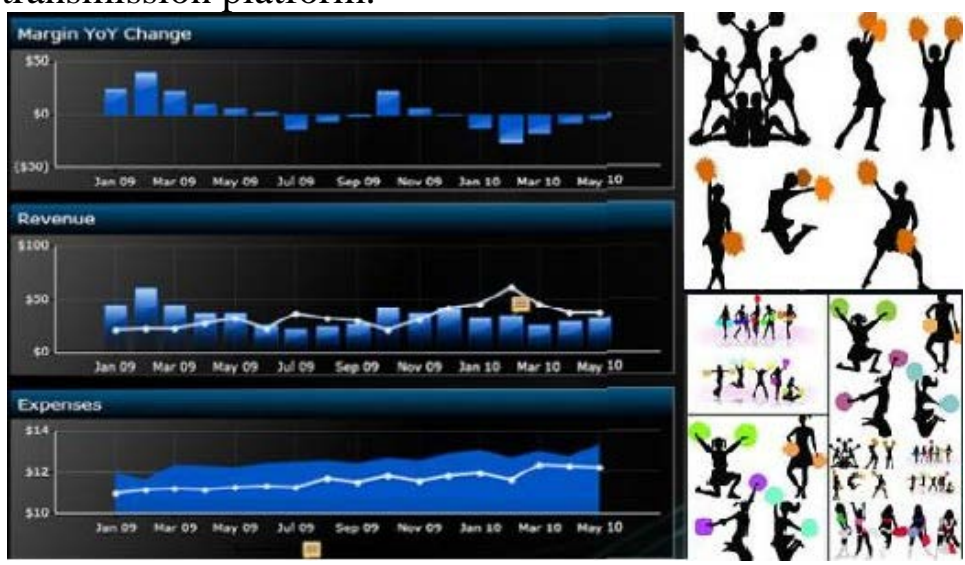

Fig.5: Aerobics movement art data analysis

Figure 5 shows the art analysis process of the aerobics movement. As shown in Figure 5, the aerobics continuous action carries out discrete decomposition and displays the display operation 
data in the curve visualization window, the data will be recorded, and then it is processed by lowpass filtering algorithm, finally we can obtain the aerobics art analysis map as shown in Figure 6. Image signal
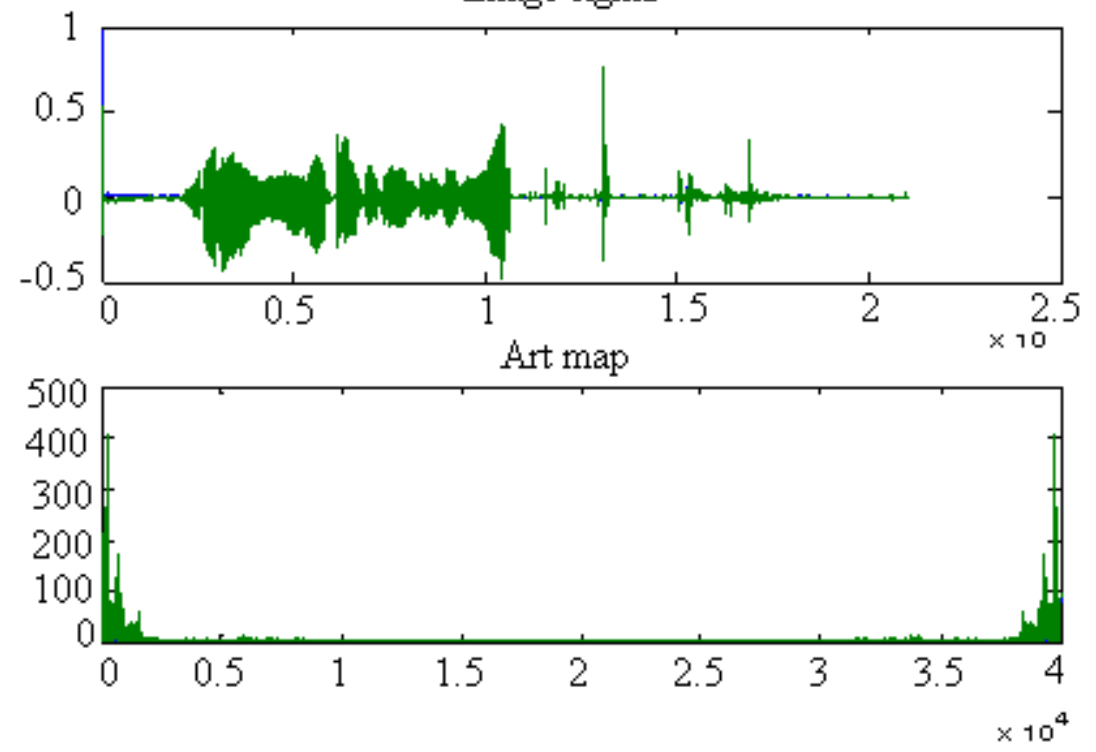

Fig.6: Aerobics art map analysis

Figure 6 shows the final result of gymnastics artistic analysis, including image signal and art atlas before and after filter. The form of using art code will filter disorganized motion, finally we retain good artistic effect image, which will realize the construction function of the aerobics art material database.

\section{Summary}

(1) The continuous video signal aerobics images are used to discrete. Using calculus filter, combined with Fourier transform and Laplasse transform, this paper designs the low-pass filtering algorithm of aerobics image, and using the multidimensional method carries out programming design for the image processing algorithm.

(2) Using WPF and JAVA heterogeneous platform, combined with modern digital media technology, this paper designs aerobics 3D visual display platform, and using filter algorithm carries out the chaotic motion filtering, we can get the are map of aerobics movement, which improve the theory reference for the aerobics art material library.

\section{References}

[1] X.X. Ma. Research on the aesthetic forms of new media installation art - form aesthetics. Popular literature, 2011(23): 56-59.

[2] Li L.S., L.J. Fei. On the artistic dissemination characteristic and the boundaries. Art education, 2011(7): 65-68.

[3] G.P. Li. The subversion and reconstruction of the network media ecology rights relationship. Journal of Liaoning Administration Institute, 2011(12): 45-46.

[4] M.F. Huang. On art industry and its localization thinking. Ningbo radio and TV University Journal, 2011(4): 61-65.

[5] Q. Wu, A.A.Wang. Thoughts of digital art bring in the era of network. Decision exploration, 2011(7): 34-36.

[6] S.Y. Du. The significance of media and its aesthetic art. Literature review, 2012(4): 112-115.

[7] G.P. Qiu, Z.B. Lin. The new thinking of art communication in the information age. Art technology, 2011(2): 35-38.

[8] Y. Zhang. The strategy of library knowledge service from the perspective of relevance data. Journal of library, 2013(2): 75-78 
[9] X. Gao. Aesthetics research technique. Modern communication, 2011(3): 52-55

[10] J.X. Yao. Liotta De's postmodern aesthetics sublime. Nanjing social science, 2011(8): 96-99.

[11] Y.G. Guan. Reverse engineering data processing technique based on Geomagic Studio. Technology Innovation Herald, 2011(33): 39-44.

[12] Y. Liu, Z.F. Hong, J.B. Qin. Study on 3D modeling technology based on graphics and image. Digital technology and application, 2011(5): 96-99.

[13] L. Gao. The overview of information visualization. Industry and Technology forum, 2011(8): 121-122.

[14] J. Qiao, J.H. Guo, T.L. Lan. The 3D data model of Using the picture modeling technology reconstruction cultural relics. The protection of cultural relics and Archaeological Science, 2012(1): 67-79.

[15] M. Hua, X. Chen, W.C. Wang. The main texture and simple extraction system. Journal of computer aided design and computer graphics, 2011(1): 69-71. 